Check for

Check for

Cite as

Nano-Micro Lett.

(2021) 13:50

Received: 20 August 2020

Accepted: 13 October 2020

Published online: 5 January 2021

(C) The Author(s) 2021

\section{Efficient Catalytic Conversion of Polysulfides by Biomimetic Design of "Branch-Leaf" Electrode for High-Energy Sodium-Sulfur Batteries}

\author{
Wenyan $\mathrm{Du}^{1}$, Kangqi Shen ${ }^{2}$, Yuruo $\mathrm{Qi}^{1}$, Wei Gao ${ }^{1}$, Mengli Tao ${ }^{1}$, Guangyuan $\mathrm{Du}^{1}$, \\ Shu-juan Bao ${ }^{1}$, Mingyang Chen ${ }^{3}$, Yuming Chen ${ }^{4}$, Maowen $\mathrm{Xu}^{1}{ }^{凶}$
}

\title{
HIGHLIGHTS
}

- 3D "branch-leaf" biomimetic design is proposed for high-performance Na-S batteries.

- The conductive "branch" can ensure adequate electron and electrolyte supply with the "leaf" can catalyze the conversion of polysulfides.

- DFT calculation reveals that the Co nanoparticles can enable fast reduction reaction of the polysulfides;

- The prepared CNF-L@Co/S electrode exhibits a high initial specific capacity of $1201 \mathrm{mAh} \mathrm{g}^{-1}$ at $0.1 \mathrm{C}$ and superior rate performance.

\begin{abstract}
Rechargeable room temperature sodium-sulfur (RT Na-S) batteries are seriously limited by low sulfur utilization and sluggish electrochemical reaction activity of polysulfide intermediates. Herein, a 3D "branch-leaf" biomimetic design proposed for high performance $\mathrm{Na}-\mathrm{S}$ batteries, where the leaves constructed from Co nanoparticles on carbon nanofibers (CNF) are fully to expose the active sites of Co. The CNF network acts as conductive "branches" to ensure adequate electron and electrolyte supply for the Co leaves. As an effective electrocatalytic battery system, the 3D "branch-leaf" conductive network with abundant active sites and voids can effectively trap polysulfides and provide plentiful electron/ions pathways for electrochemical reaction. DFT calculation reveals that the Co nanoparticles can induce the formation of a unique $\mathrm{Co}-\mathrm{S}-\mathrm{Na}$ molecular layer on the Co surface, which can enable a fast reduction reaction of the polysulfides. Therefore, the prepared "branch-leaf" CNF-L@Co/S electrode exhibits a high initial specific capacity of $1201 \mathrm{mAh} \mathrm{g}^{-1}$ at $0.1 \mathrm{C}$ and superior rate performance.
\end{abstract}

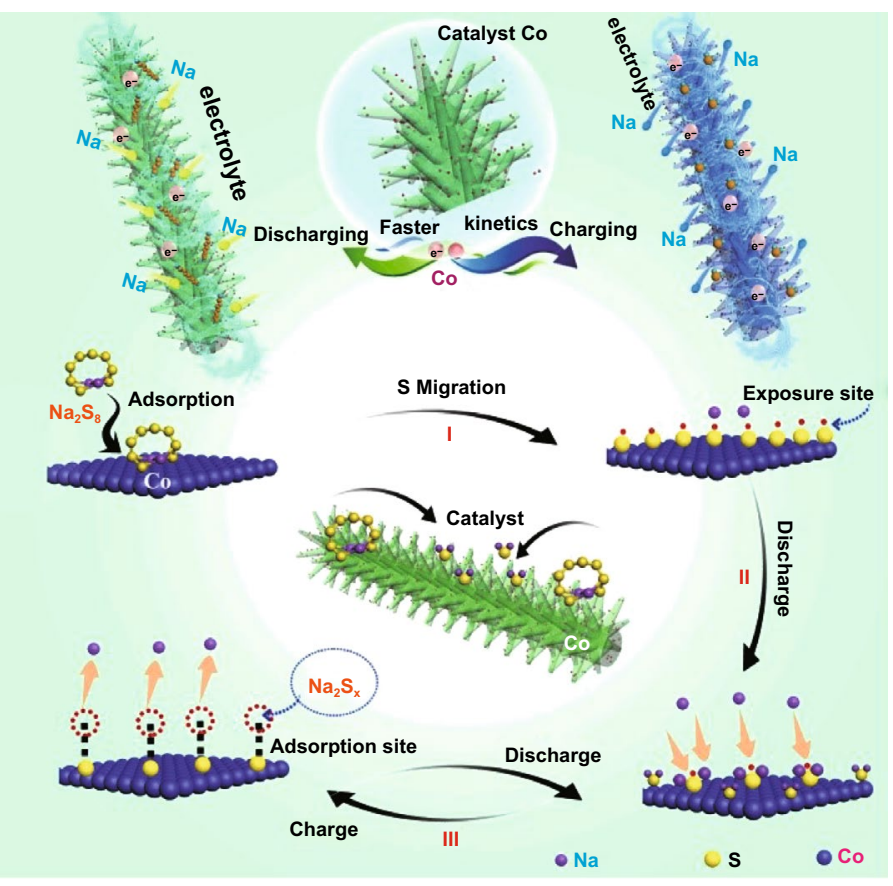

KEYWORDS Co nanoparticles; Sodium-sulfur batteries; Branch-leaf, biomimetic

Mingyang Chen, mychen@ustb.edu.cn; Yuming Chen, yumingc126@126.com; Maowen Xu, xumaowen@ swu.edu.cn

1 Key Laboratory of Luminescence Analysis and Molecular Sensing (Southwest University), Ministry of Education, School of Materials and Energy,

Southwest University, Chongqing 400715, People's Republic of China 


\section{Introduction}

To store renewable energy sources, which possess the characteristics of intermittency and randomness, rechargeable battery techniques have been widely investigated by the electrical energy storage community $[1,2]$. Among the rechargeable battery techniques, room temperature sodium-sulfur (RT $\mathrm{Na}-\mathrm{S}$ ) batteries have emerged as one of the most promising "green technique" due to their high theoretical specific capacity $\left(1675 \mathrm{mAh} \mathrm{g}^{-1}\right)$ and eco-friendly of sulfur [3, 4]. However, the intrinsic electronic/ionic insulation of sulfur and its reduction products is extremely unsatisfactory to achieve the full utilization of the active material (sulfur) [5, 6]. Moreover, the shuttling behaviors of dissolved intermediate sodium polysulfides (NaPSs) would result in poor cycling stability [7, 8].

To alleviate these drawbacks, great efforts have been devoted by researchers to design and synthesize favorable S-based cathodes with unique compositions and structures for building high performance RT Na-S batteries $[9,10]$. The most common strategy is to physically confine sulfur in various carbon materials, such as micro/mesoporous carbon, 2D graphene, hollow carbon nanotubes, and conductive polymers [11, 13]. However, such non-/weak-polar carbon materials only offer weak interactions toward polar NaPSs, which are insufficient to suppress the shuttling effect over a long lifespan, finally making against the long-term cycle stability of sulfur cathode. Therefore, other polar functional groups, such as polar metal-based compounds like oxides, nitrides, carbides, and hydroxides, have been developed as cathode hosts of sulfur to adsorb polar NaPSs by forming strong polar interaction $[14,15]$. Although these materials can strengthen the interaction between NaPSs and sulfur cathodes, most of them still suffer from low sulfur utilization, primarily due to the slow redox kinetics of polysulfides conversion, large radius of $\mathrm{Na}^{+}$, and its inferior reactivity with sulfur. The sluggish kinetics of polysulfides conversion also increase the accumulation of soluble intermediate NaPSs in the cathode area and their inevitable diffusion into the electrolyte from the cathode to anode forms insoluble solid $\mathrm{Na}_{2} \mathrm{~S}_{2} / \mathrm{Na}_{2} \mathrm{~S}$ [16, 17]. Recently, the catalysis of the polysulfide conversion has been reported as an effective method to fundamentally address the loss of sulfur by accelerating the redox kinetics of polysulfides conversion [18]. Since "polysulfide catalysis" is a relatively new concept, the exploration of more catalyst candidates with high activity and low cost, as well as a more efficient catalytic polysulfide conversion is a priority area of research. Currently, various transition metals are widely used to speed up the electrochemical reaction rates, according to results from Li-S battery [19-23]. For instance, wang's group reported that monodisperse cobalt atoms embedded in grapheme can trigger the surface-mediated reaction of Li polysulfides since the $\mathrm{Co}-\mathrm{N}-\mathrm{C}$ coordination center serves as a bifunctional electrocatalyst. This conception leads to the rapid formation of short-chain polysulfide and ultimately $\mathrm{Li}_{2} \mathrm{~S}$ and can also enhance the conductivity of the cathode [24]. A similar concept has been demonstrated in RT Na-S batteries. Yang's group reported that $\mathrm{Cu}$ nanoparticles loaded in mesoporous carbon can be utilized to immobilize sulfur and polysulfides, while a novel $\mathrm{Cu}$ foam current collector is able to activate sulfur electroactivity. In our group, we designed and synthesized a 3D host consisting of carbon fiber concatenated nickel hollow spheres for accelerating the electrochemical kinetics of RT Na-S batteries. Each nickel atom exhibits a significant catalytic effect in the conversion of polysulfide to accelerate the reaction kinetics of liquid intermediates, finally alleviating the shuttle effect to some extent. Similarly, Zhang et al. reported a highly effective sulfur host with atomic Co (including SA Co and Co clusters) supported in the micropores of hollow carbon (HC) nanospheres as a cathode for RT Na-S batteries [15]. In their study, they have fundamentally studied the mechanism for the improvement of electrochemical performance and found that the maximized atomic utilization could optimize the multiple functions of cobalt metal enhancing sulfur conductivity, activating sulfur reactivity, and immobilizing sulfur and sodium polysulfides [15]. The results of the latter work also show that the metal Co nanoparticles possess metallic characteristics and even super catalytic activity, which largely beneficial to facilitate the redox reaction kinetics and increase the sulfur utilization efficiency for Na-S batteries [24, 25]. Despite such promising results, the charge-discharge mechanism of cobalt as a catalyst in RT Na-S batteries is not clear and needs further investigation. In addition, traditional sulfur cathode,

\footnotetext{
2 Beijing Computational Science Research Center, Beijing 100193, People's Republic of China

3 Center for Green Innovation, School of Materials Science and Engineering, University of Science and Technology Beijing, Beijing 100083, People's Republic of China

4 College of Environmental Science and Engineering, Fujian Normal University, Fuzhou 350007, People's Republic of China
} 
which is prepared based on slurry-casting technique, with large amounts of inactive materials (including conductive agent, polymer binder, and metal current collectors) would significantly decrease the energy density of batteries [27]. In contrast, self-supported binder-free cathode hosts, such as carbon nanofibers can avoid this disadvantage, thus have attracted much interest from researchers. Electrospinning is one strategy to effectively produce freestanding nanofibers with excellent mechanical integrity and outstanding conductivity [28, 29]. Therefore, it is rational, yet challenging, to introduce catalyst into a free-standing sulfur electrode to maximize the various functions of a polarized sulfur host and achieve extraordinary performance for RT Na-S batteries. Metal-organic framework(MOFs) are often used as a sacrificial template to convert as-prepared hierarchical structures into nanoporous carbon while retaining the desirable properties. In addition, nitrogen-containing MOFs will yield $\mathrm{N}$-doped porous carbons upon thermal carbonization, which could greatly enhance the catalytic activity of porous carbons for electrochemical storage applications. The zeolitic imidazolate framework (ZIF) is type of MOF with a multiple structure containing mesoporous and microporous pore sizes. ZIF is particularly of interest due to its simple fabrication process and nitrogen-containing precursor [30, 31].

The cooperation of branches and leaves to convert solar energy into biological energy through photosynthesis is one of the most effective catalytic systems found in nature. In this system, branches continuously supply water and nutrients while leaves absorb solar energy simultaneously; both are critical for efficient photosynthesis (Fig. 1). Remarkably, the electrochemical catalytic process is similar to photosynthesis observed in trees. In electrochemical catalysis, active sites accelerate the reaction rate, while current collectors are responsible for the rapid transmission of electrons. Motivated biologically, a "branch-leaf' cathode host was designed in this work.

Herein, in order to achieve high performance RT Na-S batteries with increased energy density, a self-support "branch-leaf" structural cathode (CNF-L@Co/S) was designed and synthesized by integrating well-conductive carbon fibers as the "branches" via electrospinning technique and carbon coated Co nanoparticles as the "leaves". The conductive "branch"can ensure adequate electron and electrolyte supply and physically confine S/NaPSs. The carbon coated Co "leaves" can anchor polysulfides and then catalyze their conversion. Finally, attributed to the synergistic effect of the "branch-leaf" structure, the as-prepared CNF-L @ Co/S cathode exhibits a high reversible capacity of $1201 \mathrm{mAh} \mathrm{g}^{-1}\left(602.5 \mathrm{Wh} \mathrm{Kg}^{-1}\right)$ at $0.1 \mathrm{C}$, excellent cycling stability, and superior rate capability. In addition, DFT calculations demonstrate that a unique $\mathrm{Co}-\mathrm{S}-\mathrm{Na}$ molecular layer formed on the surface of Co nanoparticles, which can enable rapid redox reaction of the polysulfides.

\section{Experimental Section}

\subsection{Materials and Methods}

\subsubsection{Preparation of CNF Matrix}

PAN nanofibers were fabricated through an electrospinning technique. A precursor solution was first prepared by dissolving $1000 \mathrm{mg}$ PAN (MW=150,000) into $10 \mathrm{~mL} \mathrm{N,N-}$ dimethylformamide (DMF) with vigorous stirring, forming a pale yellow viscous homogenous solution. Then, the solution was sucked into a $10 \mathrm{~mL}$ plastic syringe with a needle and positioned $18 \mathrm{~cm}$ away from the collector. When a high voltage of $30 \mathrm{kV}$ was applied to the system, PAN nanofibers were generated and collected with a feeding rate of $0.0008 \mathrm{~mm} \mathrm{~s}^{-1}$. The as-spun white nanofiber films were stabilized at $60^{\circ} \mathrm{C}$ for $12 \mathrm{~h}$ in a vacuum oven.

\subsubsection{Preparation of Leaf-like Zeolitic Imidazolate Framework (ZIF-L) on CNF}

The CNF/ZIF-L precursor was synthesized using a solution method. First, $582 \mathrm{mg} \mathrm{Co}\left(\mathrm{NO}_{3}\right)_{2} \cdot 6 \mathrm{H}_{2} \mathrm{O}$ and $1300 \mathrm{mg}$ 2-methylimidazole (2-MIM) were dissolved in $40 \mathrm{~mL}$ deionized water. Then, cobalt nitrate solution was quickly added to the 2-MIM solution and mixed under agitation for $1 \mathrm{~min}$ at room temperature to obtain a purple solution. Next, a piece of PAN nanofiber film was directly immersed in the mixture solution and aged for $50 \mathrm{~min}$ under room temperature. After rinsing the sample with deionized water and allowing it to air dry, a purple ZIF on fiber film was obtained.

\subsubsection{Preparation of CNF-L@Co/S Composite}

The obtained purple ZIF on fiber film was placed in a porcelain boat and first annealed at $350{ }^{\circ} \mathrm{C}$ for 20 min with a heating 


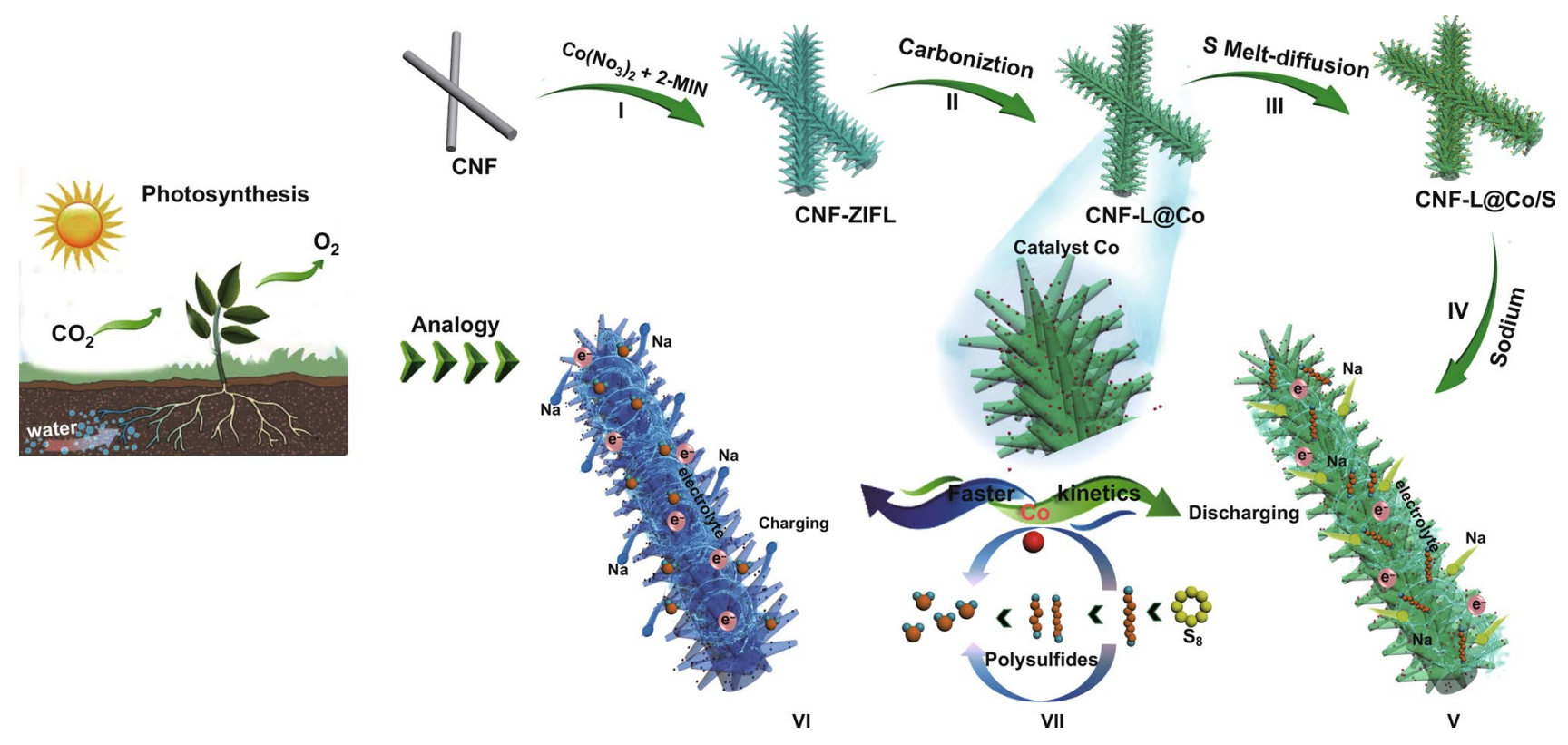

Fig. 1 Schematic of the preparation of CNF-L@Co/S and the catalytic mechanism of CNF-L@Co/S in RT Na-S batteries

rate of $2{ }^{\circ} \mathrm{C} \mathrm{min}^{-1}$. Then, the temperature increased to $800^{\circ} \mathrm{C}$ at a rate of $5{ }^{\circ} \mathrm{C} \mathrm{min}^{-1}$ maintained for $2 \mathrm{~h}$ under the protection of Ar atmosphere to obtain the CNF-L@Co compound. Finally, the sample pressed into small round disks with size of $1 \times 1 \mathrm{~cm}^{2}$ using a table press. For uniform sulfur impregnation, the prepared CNF-L@Co compound was completely soaked in the $\mathrm{S} / \mathrm{CS}_{2}$ solution $\left(10 \mathrm{mg} \mathrm{mL}^{-1}\right)$ using a pipette then dried in an airing chamber. Finally, the composite was heat-treated at $155^{\circ} \mathrm{C}$ around the melting point of sulfur for $12 \mathrm{~h}$ to obtain the uniform composite. For comparison, to remove the Co species, the acquired black electrodes were then immersed in $6 \mathrm{M}$ $\mathrm{HCl}$ aqueous solution via a hydrothermal reaction at $130{ }^{\circ} \mathrm{C}$ for $2 \mathrm{~h}$. After being washed with deionized water to remove the acid, CNF-L@Co/S was successfully converted into CNF$\mathrm{L} / \mathrm{S}$. CNF/S film was also produced following same method but without ZIF.

\subsection{Electrochemical Measurements}

The free-standing CNF-L@Co/S and CNF/S films were cut into $10 \mathrm{~mm}$ disks and directly used as electrodes without any other additives. The electrochemical test conditions were the same as those in our previous paper [39]. $1 \mathrm{M}$ of sodium perchlorate $\left(\mathrm{NaClO}_{4}\right.$, Sigma Aldrich, $\left.>98 \%\right)$ in a mixture of ethylene carbonate/diethyl carbonate (EC/DEC, 1:1 v/v\%) was used as the electrolyte in a dosage of $90 \mathrm{~mL}$. The total weight of the cathode was $-2.5 \mathrm{mg}$. The specific capacities were estimated based on the loading of sulfur in the cathodes $\left(1-1.2 \mathrm{mg} \mathrm{cm}^{-2}\right)$.

\section{Results and Discussion}

\subsection{Synthesis and Characterization}

Motivated biologically, a "branch-leaf" cathode host for Na-S batteries was designed in this work. The "branches" consisting of uniform carbon nanofibers (CNF) were prepared by simple electrospinning of PAN (polyacrylonitrile Fig. 1). Then, a uniform shell of ZIF with leaf-like morphology was formed on the PAN nanofibers through a solution deposition method at room temperature (Fig. 1 I). To preserve morphology and achieve high electronic conductivity simultaneously, the asprepared CNF-ZIF composite was treated through a two-step carbonization process, during which the "leaves" were formed by the accumulation of carbon coated Co nanoparticles (the final product is marked as CNF-L@Co in Fig. 1 II). Finally, sulfur was successfully impregnated into the CNF-L@Co composite (denoted as CNF-L@Co/S) by a melt-diffusion process (Fig. 1 III). 
In the as-proposed unique "branch-leaf" structure, the catalytic "leaves" (Co) on the highly conductive "branches" (CNF) have been constructed as $\mathrm{Na}-\mathrm{S}$ batteries cathode. As shown in Fig. 1 VII, the internal interwoven CNF "branch" with plentiful pores and satisfactory electronic conductivity can supply transmission channels for the electrolyte/electron, while the external "leaves" with abundant Co active sites can effectively anchor and convert sodium polysulfides. Therefore, the bioinspired "branch-leaf" structure CNF-L@Co/S appears highly promising in improving the electrochemical properties of RT $\mathrm{Na}-\mathrm{S}$ batteries.

As shown in Fig. S1, the diameter of CNFs was determined to be $400 \mathrm{~nm}$. The closely interwoven CNFs are beneficial for the transport of electrons and the infiltration of electrolyte, hence promoting electrochemical reactions. Figure $2 \mathrm{a}$, b show that the carbon nanofibers are uniformly covered by Co nanoparticles forming leaf-like architectures. The FESEM image in Fig. 2c indicates that the length and thickness of the "leaf" are about 800 and $100 \mathrm{~nm}$, respectively. The TEM image in Fig. 2d demonstrates that the Co nanoparticles are uniformly distributed in the ZIF-derived carbon and the diameter of them is less than $18 \mathrm{~nm}$. The obvious fringe with a lattice spacing about $0.20 \mathrm{~nm}$ in Fig. 2e corresponds to the (111) lattice plane of Co, indicating the successful transformation of ZIF to Co nanoparticles. In addition, Fig. 2e also shows that the thickness of the carbon layer on Co nanoparticles is around $2 \mathrm{~nm}$. The TGA curve reveals that the content of Co in the CNF-L@Co is about $14.69 \%$ (Fig. S3). The X-ray mapping images in Fig. S4 imply that all elements $(\mathrm{C}, \mathrm{N}$, and $\mathrm{Co})$ are evenly scatted within the as-prepared composite. X-ray photoelectron spectroscopy (XPS) was employed to study the chemical composition and the oxidation state of the as-prepared CNF-L@Co. As shown in Fig. S5, the N 1 s spectrum displays three strong peaks at binding energies of 401.38, 399.09, and $397.18 \mathrm{eV}$, corresponding to graphitic-N, pyrrolic-N, and pyridinic-N, respectively [32]. The pyridinic-N and pyrrolic- $\mathrm{N}$ have been reported to increase the pseudocapacitance, while graphitic-N increases the overall conductivity. Moreover, the peaks in the $\mathrm{C} 1 \mathrm{~s}$ spectrum at $283.88,285.08$, and 286.68 can be assigned to $\mathrm{C}=\mathrm{C} s p^{2}, \mathrm{C}-\mathrm{N} / \mathrm{C}-\mathrm{C} s p^{3}$, and $\mathrm{C}=\mathrm{O}$ species, respectively, which further confirms the $\mathrm{N}$ doping in the carbon matrix (Fig. S5a, b) [33]. In the corresponding Raman spectrum in Fig. S6, CNF-L@Co exhibits two broad peaks at $1337 \mathrm{~cm}^{-1}$ (D band) and $1575 \mathrm{~cm}^{-1}$ (G band), revealing its graphite-like microcrystalline structure, and peaks at $672 \mathrm{~cm}^{-1}$ are assigned to the ring structure of $\mathrm{Co}$. In addition, the nitrogen heteroatom substitution into the carbon matrix generates defective sites, thus increasing in the $I_{\mathrm{D}} / I_{\mathrm{G}}$ intensity $[34,35]$.

In Fig. 3d, the pristine Co 2P XPS spectrum can be deconvoluted into $\mathrm{Co}^{0}(778.88 \mathrm{eV})$ and $\mathrm{Co}^{2+}(781.60 \mathrm{eV})$ which could be derived from the surface oxidation of metallic Co [36, 37]. The Brunauer-Emmett-Teller (BET) surface area and pore volume of CNF-L@Co are $55.662 \mathrm{~m}^{2} \mathrm{~g}^{-1}$ and $0.089 \mathrm{~cm}^{3} \mathrm{~g}^{-1}$, respectively, which is beneficial for the maintenance of sulfur.

Finally, sulfur was successfully impregnated into the CNF-L@Co composite (denoted as CNF-L@Co/S) by a meltdiffusion process. The obtained freestanding CNF-L@Co/S cathode possesses high flexibility and can be directly used as binder-free cathode for RT Na-S batteries. As shown in Fig. 2f, prominent diffraction peaks of orthorhombic sulfur are detected in the CNF-L@Co/S composite accompanied by three strong peaks of Co (JCPDS No. 89-4307), confirming the successful incorporation of sulfur. Structural characterization of the flexible CNF-L@Co/S electrode was also investigated by FESEM and TEM. As shown in Fig. S7, the CNF-L@Co/S composite maintains the original morphology after sulfur loading, and no excess sulfur block can be observed on the surface of the material. The TEM images in Fig. S7d demonstrate that most of the interior space of CNF-L@Co/S become darker, further indicating the sulfur was successfully diffused into the substrate, while a small amount of void space is still left, which could buffer the volume expansion during the discharge/charge process. In addition, the BET surface area and pore volume are substantially reduced to $11.323 \mathrm{~m}^{2} \mathrm{~g}^{-1}$ and $0.028 \mathrm{~cm}^{3} \mathrm{~g}^{-1}$ (Fig. S7f), respectively, revealing the successful accommodation of sulfur into the pores of the CNF-L@Co material. The TGA analysis in Fig. S7c directly confirms that the sulfur content of CNF-L@Co/S is about 45\%. The elemental mapping images in Fig. $2 \mathrm{~g}-\mathrm{k}$ verify the homogenous distribution of $\mathrm{S}$, $\mathrm{Co}$, and $\mathrm{C}$, indicating the uniform chemical composition of the CNF-L@Co/S composite material. The stable connection between the "branches" (CNF) and "leaves" (carbon coated Co nanoparticles) supports the efficient electron migration between the conductive carbon substrate and active surface feasible, enabling rapid interfacial reactions. It is worth noting that the mechanical flexibility (shown in Fig. 2i) of the asobtained CNF-L@Co/S supports its potential as self-supported electrodes for $\mathrm{Na}-\mathrm{S}$ batteries. 

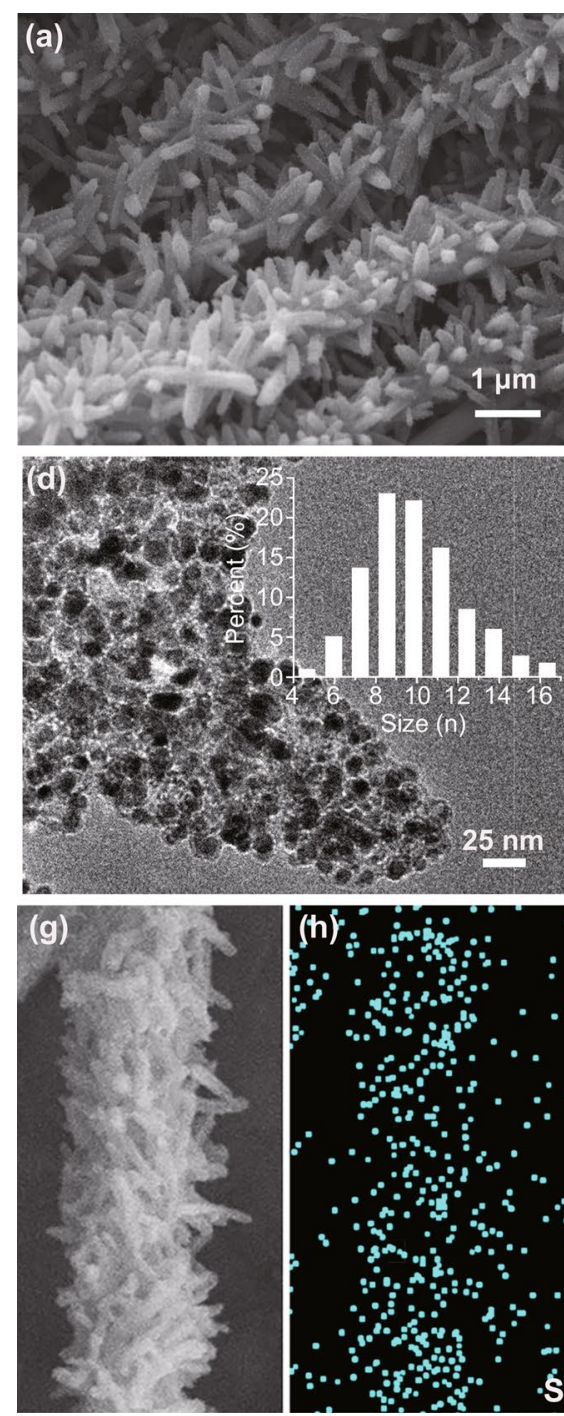
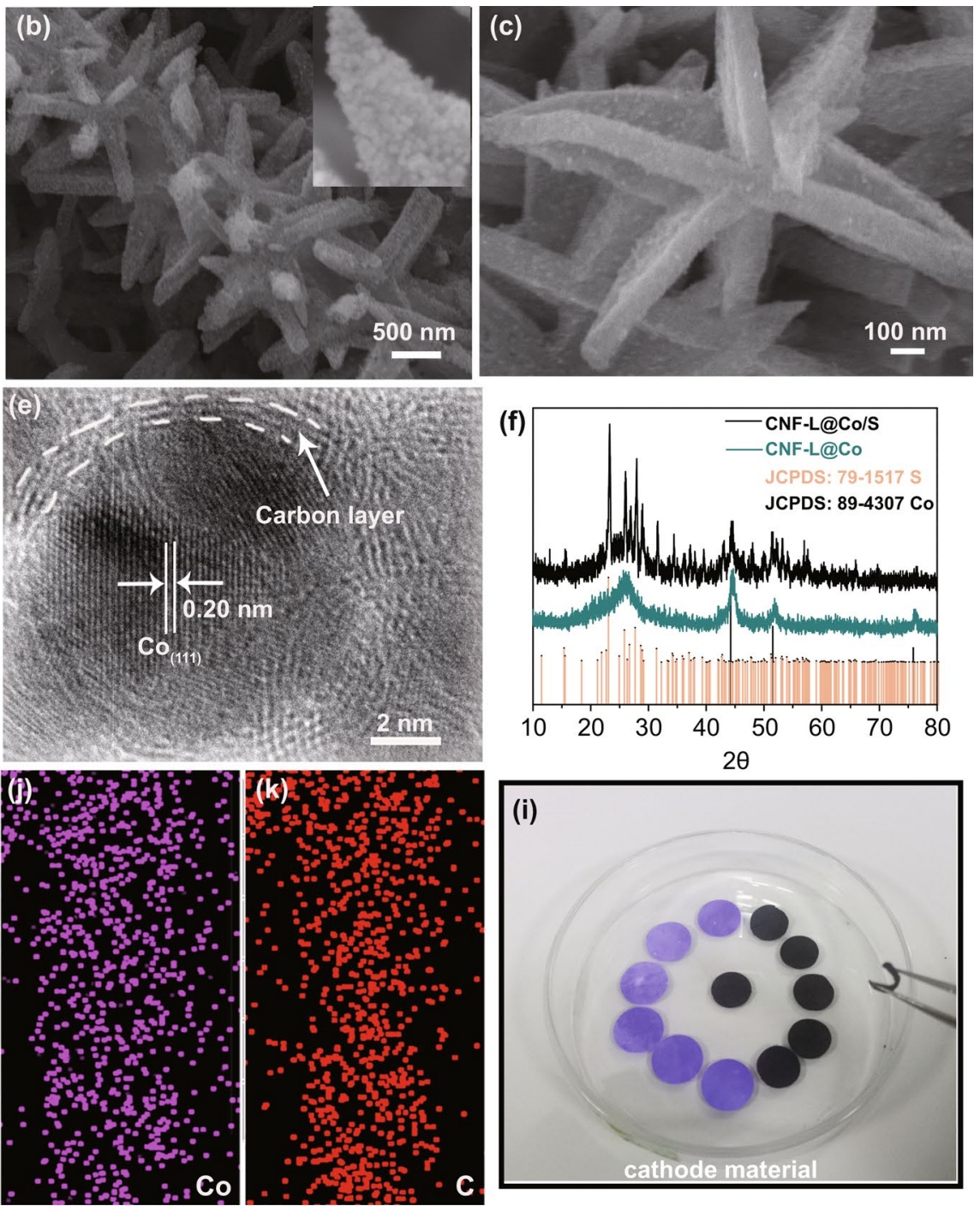

Fig. 2 Characterization composites. a-c FESEM images of CNF-L@Co, d, e TEM images of CNF-L@Co; $\mathbf{f}$ XRD pattern of CNF-L@Co and CNF-L@Co/S; g-k EDS elemental mappings of CNF-L@Co/S, and i flexible films of CNF-L@Co/S

\subsection{Electrocatalytic Activity Investigation}

The thickness of the free-standing film electrode material is $40.63 \mu \mathrm{m}$ (Fig. S12a). To demonstrate the effect of the advanced "branch-leaf" structure, electrochemical performances of CNF-L@Co/S and bare CNF/S composite were evaluated by RT Na-S batteries. Figure 3a shows galvanostatic discharge/charge profiles of the CNF-L@Co/S and $\mathrm{CNF} / \mathrm{S}$ electrodes at $0.1 \mathrm{C}$. In Fig. 3a, it can be observed that CNF-L@Co/S exhibits a more prominent discharge plateau and reduced polarization (compared to $732 \mathrm{vs} 1138 \mathrm{mV}$ of $\mathrm{CNF} / \mathrm{S}$ ). The existence of metallic Co is indeed helpful to dynamically accelerate the electrochemical reactions of sodium polysulfides. Figure. S8a illustrates the chemical interaction on the Co interface. Figure. S8b presents the cyclic voltammogram (CV) curves of both samples at a scan rate of $0.1 \mathrm{mV} \mathrm{s}^{-1}$. During the first scan, a pair of redox peaks at 1.8/1.04 $\mathrm{V}$ corresponds to the conversion of long-chain polysulfides $\left(\mathrm{Na}_{2} \mathrm{~S}_{x}, x=4-8\right)$ to short-chain sodium polysulfides $\left(\mathrm{Na}_{2} \mathrm{~S}_{2} / \mathrm{Na}_{2} \mathrm{~S}\right)$ and reversely the oxidation of $\mathrm{Na}_{2} \mathrm{~S}$ $[13,38]$.

Compared with CNF/S, the CNF-L@Co/S electrode manifests a smaller potential hysteresis in its voltage profile as well as smaller gaps between the paired redox peaks in the $\mathrm{CV}$ 

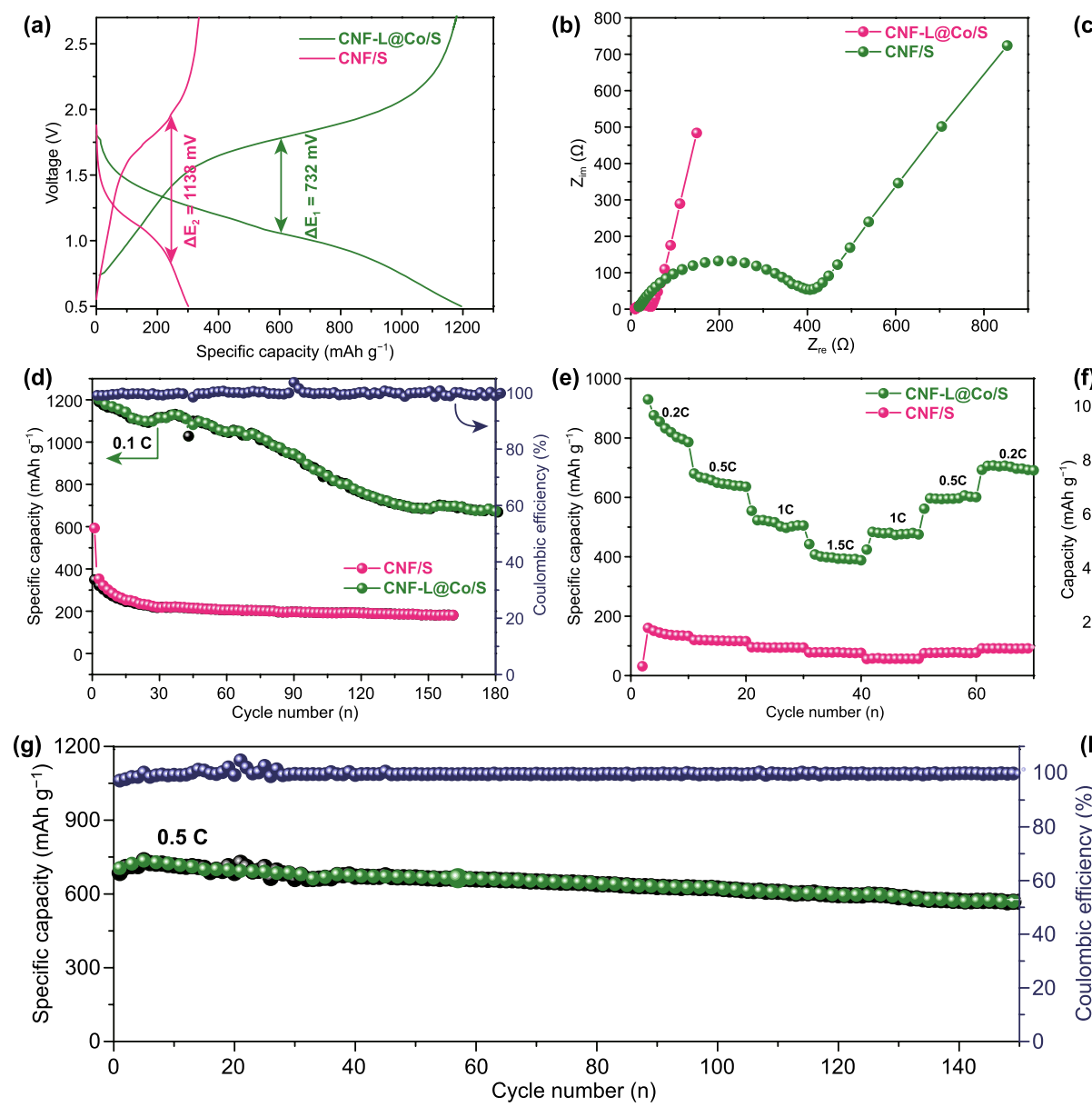
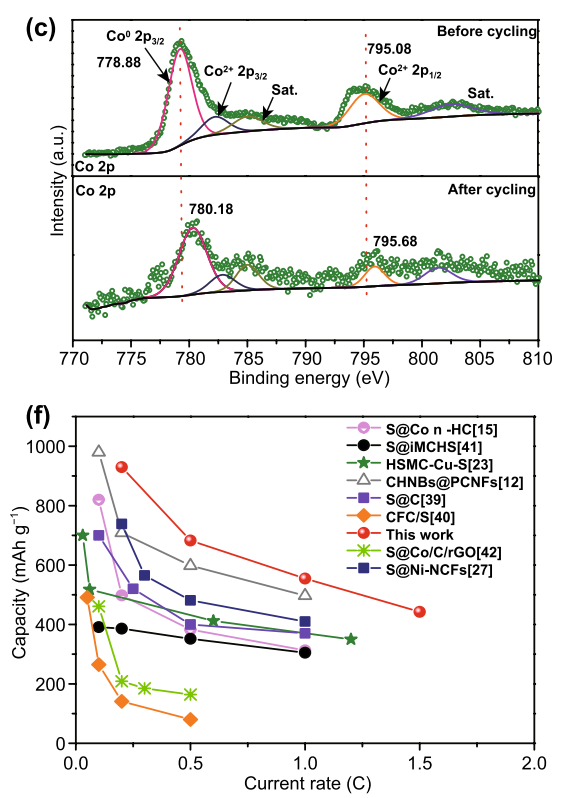

(h)

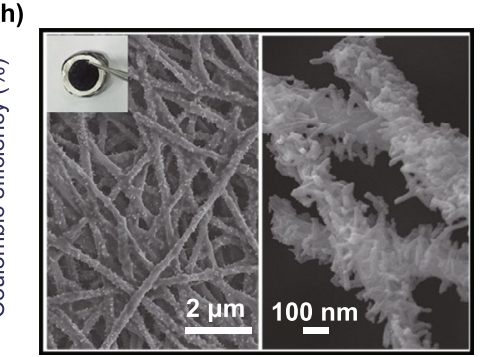

Fig. 3 a Comparison of discharge/charge curves. b Comparison of electrochemical impedance spectra. c High-resolution XPS spectra of Co $2 p$ before and after cycling. d Cycling performance and Coulombic efficiency of CNF-L@Co/S and CNF/S at $0.1 \mathrm{C}$. e Rate performance of CNF-L@Co/S and CNF/S. f Comparison of the rate capabilities with previously reported RT Na-S batteries. $\mathrm{g}$ Long-term cycling stability of CNF-L@Co/S at $0.5 \mathrm{C}$, and h FESEM images of the CNF-L@Co/S composite after cycling

curves in Fig. S9, which serves as pronounced evidence for the catalytic effect of Co nanoparticles. Moreover, such kinetic improvement of the CNF-L@Co/S electrode was further supported by electrochemical impedance spectroscopy (EIS) analysis. As shown in Fig. 3b, the charge-transfer resistance of CNF-L@Co/S electrode is much a smaller than that of the $\mathrm{CNF} / \mathrm{S}$ electrode (30 vs $198 \Omega$ ), indicating its faster charger transfer behavior [39]. Both the doped $\mathrm{N}$ and added Co nanoparticles can help improve the electronic conductivity of CNF and the chemical affinity of the as-prepared electrode with NaPSs. As displayed in Fig. 3c, the two characteristic peaks of $\mathrm{Co}$ (at 778.88 and $795.08 \mathrm{eV}$ in pristine electrode, attributed to the metallic $\mathrm{Co}$ and oxidized $\mathrm{Co}^{2+}$ ) both shift to higher binding energies ( -780.18 and $-795.68 \mathrm{eV}$ ) after cycling, indicating the strong chemical interaction between Co and NaPSs [40].
Comparison of cycling performance of the two electrodes is provided in Fig. 3d. The CNF-L@Co/S electrode shows a higher initial capacity than $\mathrm{CNF} / \mathrm{S}$ at $0.1 \mathrm{C}$ (1201 vs $349 \mathrm{mAh} \mathrm{g}^{-1}$ ), confirming a more efficient polysulfide redox reaction. The unique "leaf-branch" structure ensures effective utilization of the Co "leaf" during electrocatalytic reactions and fast electron transfer by the porous CNF "branch", which plays a key role in improving the overall electrochemical performance. Moreover, the improved redox kinetics in $\mathrm{CNF}-\mathrm{L} @ \mathrm{Co} / \mathrm{S}$ is further revealed by its superior rate performance shown in Fig. 3e. The electrode with CNF-L@Co/S delivers a high discharge capacity of about $929.8 \mathrm{mAh} \mathrm{g}^{-1}$ at $0.2 \mathrm{C}$, indicating greater sulfur utilization. Even at increased current rate of $1.5 \mathrm{C}$, the capacity of the CNF-L@Co/S electrode still remains as high as $442.7 \mathrm{mAh} \mathrm{g}^{-1}$, which five times 
of the CNF/S electrode $\left(77.7 \mathrm{mAh} \mathrm{g}^{-1}\right)$. Notably, when the current rate was reduced to $0.2 \mathrm{C}$, the discharge capacity of the CNF-L@Co/S electrode recovered to $704.6 \mathrm{mAh} \mathrm{g}^{-1}$, demonstrating its outstanding stability under high current rates. As shown in Fig. S10a, after etching $\mathrm{Co}$ by $\mathrm{HCl}$ aqueous solution, the overall morphology of the material remained unchanged, leaving abundant macropores on the surface of CNF-L (Fig. S10b). Furthermore, the supplementary electrochemical properties of the CNF-L/S electrode in Fig. S10c, $\mathrm{d}$, The CNF-L/S electrodes exhibit inferior cycle capabilities relatively of $486.5 \mathrm{mAh} \mathrm{g}^{-1}$ at $0.1 \mathrm{C}$ in Fig. S10c. Moreover, it is also suffer slower rate capacity compared to CNF-L@ $\mathrm{Co} / \mathrm{S}$ (Fig. 5d), In addition, the average discharge capabilities of the CNF-L/S at 0.1, 0.2, 0.5, 1, and $1.5 \mathrm{C}$ were 678.5, 657.7, $593.3,507.8$, and $412.1 \mathrm{mAh} \mathrm{g}^{-1}$, respectively. The results suggest that the overall electrochemical performance of the CNF-L@Co/S electrode is superior than that of both CNF/S and CNF-L/S electrodes, which confirms the synergistic effect of Co "leave" and CNF "branch" in improving the redox kinetics of polysulfides conversion.

A comparison of the rate capability with that in the state-ofthe-art literatures is presented in Fig. $3 \mathrm{f}$ and Table S1, which indicates that the rate capability of the prepared CNF-L@Co/S electrode outperforms the majority of reported electrodes [12, $15,23,25,41-44]$. Figure $3 \mathrm{~g}$ shows the long-term cycling performance of the electrode at $0.5 \mathrm{C}$. The $\mathrm{CNF}-\mathrm{L} @ \mathrm{Co} / \mathrm{S}$ electrode exhibits a high discharge capacity of $736.8 \mathrm{mAh} \mathrm{g}^{-1}$ at the fifth cycle and maintains at $538 \mathrm{mAh} \mathrm{g}^{-1}$ after 150 cycles with a low decay rate of $0.17 \%$ per cycle. Even at a rather high current rate of $2 \mathrm{C}$ (Fig. S11), CNF-L@ Co/S still delivered a high initial discharge capacity of $637.3 \mathrm{mAh} \mathrm{g}^{-1}$, which stabilized at $225.7 \mathrm{mAh} \mathrm{g}^{-1}$ after 1000 cycles. In addition, the shape and structure of the CNF-L@Co/S can be well retained after cycling (Fig. 3h). These results cooperatively manifest the high sulfur utilization and fast sulfur redox reaction via strong electrocatalytic Co in the "branch-leaf" CNF-L@Co/S electrode. To further validate the advantages of the CNF-L@ $\mathrm{Co} / \mathrm{S}$ cathode, we compared its structure and electrochemical activity with published studies about Co catalytic effect in RT Na-S batteries as shown in Table $\mathrm{S} 2[15,27,45]$. It is worth mentioning that the CNFs contribute to the little capacity under the same conditions so it was negligible, as displayed in Fig. S12.

The above results demonstrate that the existence of metallic Co is indeed helpful to accelerate dynamically accelerate the electrochemical reactions of sodium polysulfides, which is illstrated in Fig. S8a. In situ Raman spectroscopy is usually employed to investigate the mechanism of polysulfide formation during discharge and charge. Figure $\mathrm{S} 13$ shows the in situ Raman spectra of the CNF-L@Co/S electrode during discharge from 2.4 to $0.5 \mathrm{~V}$ followed by recharging to $2.4 \mathrm{~V}$, the curves of particular interest are marked in the corresponding voltage-capacity curve as shown in Fig. S13b. For the pristine electrode, the peaks at $187.3,337.2$, and $495.04 \mathrm{~cm}^{-1}$ correspond to the characteristic peaks of $\mathrm{S}_{8}$, according to previous literature $[16,46]$. After discharged to $2.4 \mathrm{~V}$, the peak of $\mathrm{S}_{8}$ become gradually weaker, while a new peak attributable to $\mathrm{Na}_{2} \mathrm{~S}_{4}$ appeared at about $426.2 \mathrm{~cm}^{-1}$. As the discharge continued, the peak of $\mathrm{Na}_{2} \mathrm{~S}$ (at about $463.3 \mathrm{~cm}^{-1}$ ) was observed at $1.0 \mathrm{~V}$, while the intensity kept increasing until it was fully discharged to $0.5 \mathrm{~V}$. In the charge process, the characteristic peaks of $\mathrm{Na}_{2} \mathrm{~S}_{6}, \mathrm{Na}_{2} \mathrm{~S}_{2}$, and $\mathrm{Na}_{2} \mathrm{~S}_{x}$ can be distinguished at around 342 , 477 , and $698 \mathrm{~cm}^{-1}$, respectively. After charged to $2.8 \mathrm{~V}$, the peaks of both $\mathrm{S}$ and deposited $\mathrm{Na}_{2} \mathrm{~S}_{2}$ can be detected, indicating that the reaction is partially reversible $[34,47]$. Therefore, the in situ Raman patterns reveal that sulfur mainly undergoes a conversion from solid $\mathrm{S}_{8}$ into soluble long-chain polysulfide $\left(\mathrm{Na}_{2} \mathrm{~S}_{n}, n=4-8\right)$ and a subsequent transformation into shortchain polysulfides into $\left(\mathrm{Na}_{2} \mathrm{~S}_{2} / \mathrm{Na}_{2} \mathrm{~S}\right)$, which is consistent with the $\mathrm{CV}$ curve (Fig. S8b). Based on the above results, it can be concluded that Co could quickly catalyze the reduction of $\mathrm{NaPSs}$ into $\mathrm{Na}_{2} \mathrm{~S}$ and thus effectively reduce the dissolution of NaPSs during cycling, contributing to excellent electrochemical performance of the electrode [48].

\subsection{DFT Calculations}

To gain further insight into how the encapsulated Co nanoparticles contribute to the fast kinetics of the CNF-L@Co electrode, DFT calculations were performed. The adsorption energy of $\mathrm{Na}_{2} \mathrm{~S}_{x}(x=1,2,4,6$, and 8) polysulfides on an $\mathrm{N}$-doped graphite slab and clean Co (111) slab was calculated (Fig. S14). Since the adsorption of $\mathrm{Na}_{2} \mathrm{~S}_{x}$ on the N-doped graphite is fairly weak, and thus $\mathrm{N}$-doped graphite will not affect the electronic structure of the $\mathrm{Na}_{2} \mathrm{~S}_{x}$ adsorbate; the $\mathrm{N}$-doped graphite adsorbed $\mathrm{Na}_{2} \mathrm{~S}_{x}$ can be considered as free molecules approximately. Comparatively, much stronger $\mathrm{Na}_{2} \mathrm{~S}_{x}$ adsorption was observed for Co (111) (Fig. 4a), which can be either molecular or dissociative. Among the different types of polysulfides, $\mathrm{Na}_{2} \mathrm{~S}$ and $\mathrm{Na}_{2} \mathrm{~S}_{2}$ favor the molecular adsorption on clean Co (111), whereas $\mathrm{Na}_{2} \mathrm{~S}_{4}, \mathrm{Na}_{2} \mathrm{~S}_{6}$, and $\mathrm{Na}_{2} \mathrm{~S}_{8}$ favor 

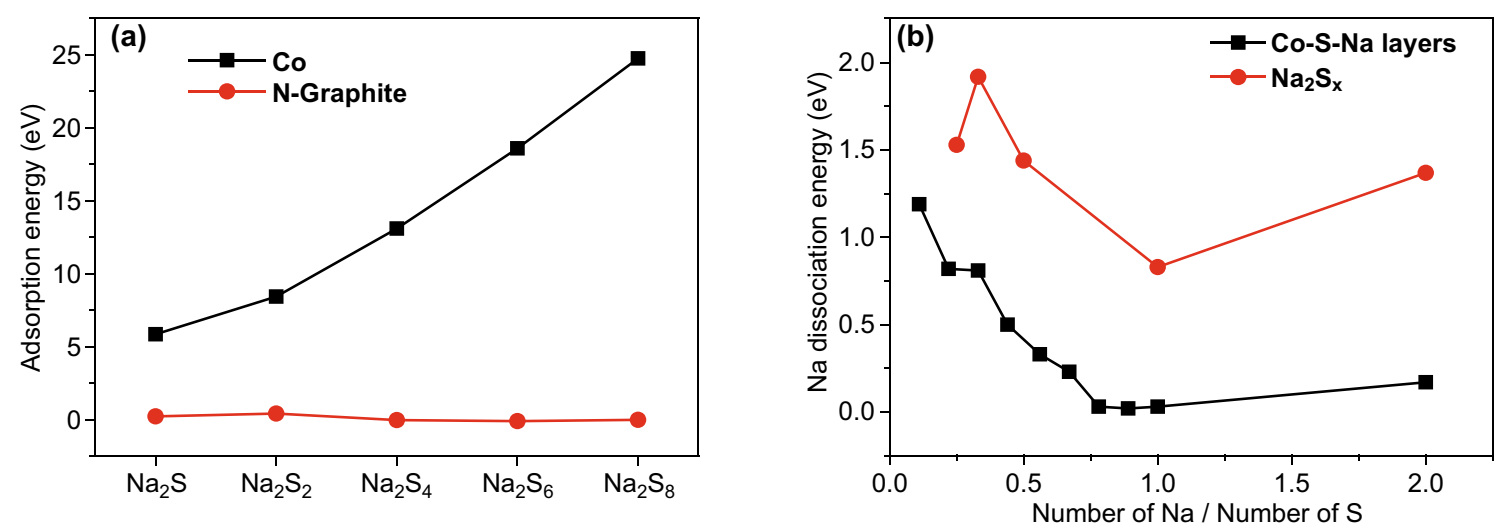

(c)

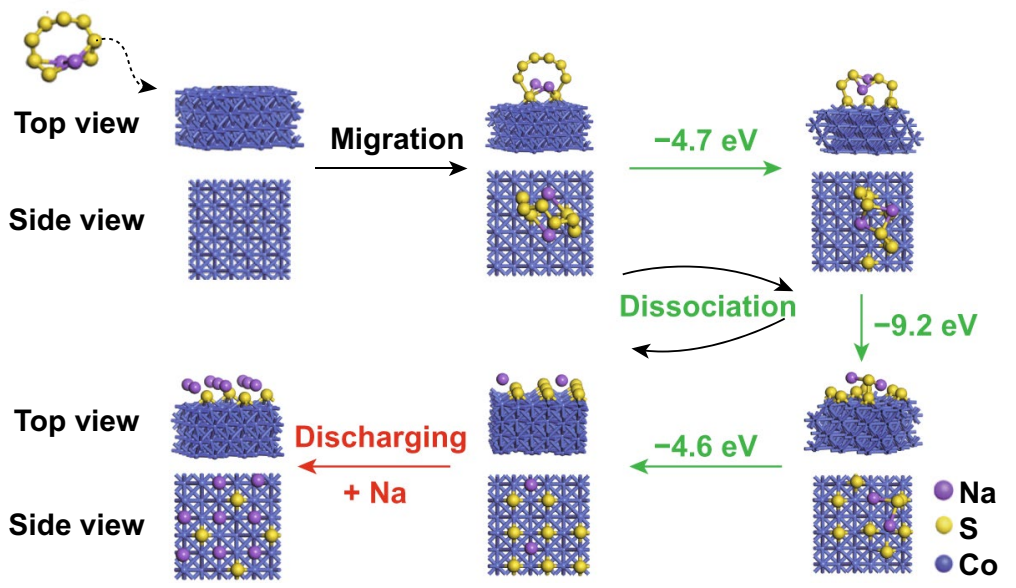

Fig. 4 a Calculated adsorption energy (eV) for the most favorable adsorption configuration of $\mathrm{Na}_{2} \mathrm{~S}_{x}$ at Co (111) surface and $\mathrm{N}$-doped graphite at the DFT level. b Dissociations for the layered Co-S-Na structures and free $\mathrm{Na}_{2} \mathrm{~S}_{x}, x=1-8$. c Conversion between the molecularly adsorbed $\mathrm{Na}_{2} \mathrm{~S}_{8}$ to the dissociatively adsorbed $\mathrm{Na} 2 \mathrm{~S} 8$ at the $\mathrm{Co}(111)$ surface via $\mathrm{S}$ migration and embedded sodium

the dissociative adsorption. The molecularly adsorbed $\mathrm{Na}_{2} \mathrm{~S}_{x}$ remains intact and forms strong $\mathrm{Co}-\mathrm{S}$ bonds with the surface. In the dissociative adsorption of $\mathrm{Na}_{2} \mathrm{~S}_{x}$, the $\mathrm{S}$ atoms of the molecular $\mathrm{Na}_{2} \mathrm{~S}_{x}$ migrate onto the Co surface and become dispersed surface $\mathrm{S}$ with $\mathrm{Co}-\mathrm{S}$ bonding (Fig. S15).

In order to better comprehend the process of the dissociative adsorption process, we took $\mathrm{Na}_{2} \mathrm{~S}_{8}$ as a typical example. In the dissociative adsorption of $\mathrm{Na}_{2} \mathrm{~S}_{8}$, the $\mathrm{S}$ atoms in the molecular $\mathrm{Na}_{2} \mathrm{~S}_{8}$ migrate onto the Co surface and become gradually dispersed surface single $\mathrm{S}$. The DFT calculation shows that such $\mathrm{Na}_{2} \mathrm{~S}_{8}$ decomposition over Co involves a series of exothermal steps $(\Delta \mathrm{H}=-4.7,-9.2$, and $-4.61 \mathrm{eV}$, Fig. 4c) and thus is thermally favorable. It is noted that, in the most favorable adsorption configurations for all $\mathrm{Na}_{2} \mathrm{~S}_{x}$, all $\mathrm{S}$ atoms form equal bonds with 4 surface Co atoms (the square $\mathrm{Co}_{4}$ surface structure), and none of the $\mathrm{Na}$ atoms form bonds with the Co surface (Fig. S15). The strong Co-S bonding accounts for the high adsorption strength of the $\mathrm{Na}_{2} \mathrm{~S}_{x}$ at the
Co surface. Consequently, when increasing the coverage of the surface-adsorbed $\mathrm{Na}_{2} \mathrm{~S}_{x}$, a surface double layer on top of the Co surface can be formed for all $\mathrm{Na}_{2} \mathrm{~S}_{x}$ (denoted as Co-S-Na, Fig. S15).

At full coverage, the molecularly adsorbed $\mathrm{Na}_{2} \mathrm{~S}_{2}$ appears as a monolayer (ML) of $\mathrm{S}$ and a $\mathrm{ML}$ of $\mathrm{Na}$ (each $\mathrm{Na}$ forming equal bonds with the $4 \mathrm{~S}$ atoms of the square $\mathrm{S}_{4}$ surface structure). The dissociative adsorbed $\mathrm{Na}_{2} \mathrm{~S}_{4}, \mathrm{Na}_{2} \mathrm{~S}_{6}$, and $\mathrm{Na}_{2} \mathrm{~S}_{8}$ can be viewed as a ML of $\mathrm{S}$ and a fractional ML of $\mathrm{Na}$ on top of S-ML; the molecularly adsorbed $\mathrm{Na}_{2} \mathrm{~S}$ exhibits the appearance of $1 \frac{2}{2} \mathrm{ML}$ of $\mathrm{S}$ and $1 \mathrm{ML}$ of $\mathrm{Na}$.

In order to comprehend the role of the Co-S-Na doublelayer adsorption structures, $\mathrm{Na}$ dissociation energies (the dissociated $\mathrm{Na}$ atom is considered in the solid state) for the layered $\mathrm{Co}-\mathrm{S}-\mathrm{Na}$ structures were first calculated then compared with the $\mathrm{Na}$ dissociation energies for the free $\mathrm{Na}_{2} \mathrm{~S}_{\mathrm{x}}$ molecules (Fig. $4 \mathrm{~b}$ and the related structures can be found in Fig. S16). The Co-S-Na structures have lower Na 
dissociation energy than free $\mathrm{Na}_{2} \mathrm{~S}_{x}$ molecules with the same ratio, as the strong $\mathrm{Co}-\mathrm{S}$ bonds between the Co surface and S-ML can significantly weaken the S-Na interactions. The energy difference between $\mathrm{Co}-\mathrm{S}-\mathrm{Na}$ and $\mathrm{Na}_{2} \mathrm{~S}_{x}$ with the same $\mathrm{Na}: \mathrm{S}$ is $-\mathrm{eV}$ per $\mathrm{Na}$ for most of the $\mathrm{Na}: \mathrm{S}$ range. As a consequence, the $\mathrm{Na}$ in $\mathrm{Co}-\mathrm{S}-\mathrm{Na}$ is more susceptible to reduction reactions than that in the $\mathrm{Na}_{2} \mathrm{~S}_{x}$ molecule, which leads to faster kinetics during the charge process. The $\mathrm{Na}$ dissociation energy is also related its reduction potential and charge capacity per $\mathrm{Na}$, i.e., the amount of energy (per $\mathrm{Na}$ ) can be chemically stored in $\mathrm{Co}-\mathrm{S}-\mathrm{Na}$ or $\mathrm{Na}_{2} \mathrm{~S}_{x}$ via the charge process. Co-S-Na has a lower charge capacity (by $-1 \mathrm{eV}$ per Na) than $\mathrm{Na}_{2} \mathrm{~S}_{x}$ with the same Na:S ratio. This implies, energetically, that $\mathrm{Co}-\mathrm{S}-\mathrm{Na}$ lies between Na solid and $\mathrm{Na}_{2} \mathrm{~S}_{x}$, so Co-S-Na might be an intermediate state for the interconversion between solid $\mathrm{Na}$ and $\mathrm{Na}_{2} \mathrm{~S}_{x}$. In other words, the surface of the Co nanoparticles acts as a catalyst during the charge stage.

Based on the above analyses, we propose the catalytic mechanism for Co nanoparticles induce fast reduction kinetics and enhances the battery performance (Fig. 5). The Co-S-Na structure first forms on the exposed surfaces of Co nanoparticles and acts as a catalyst site during discharge and possibly during charge. This process is predicted to be highly irreversible and thus may only occur during the initial battery runs (Fig. 5c I). During discharging process (Fig. 5c II), the oxidized $\mathrm{Na}^{+}$in the electrolyte enriches near $\mathrm{Co}$, which allows $\mathrm{Na}^{+}$to be directly absorbed onto $\mathrm{Co}-\mathrm{S}-\mathrm{Na}$ to form $\mathrm{Na}_{2} \mathrm{~S}$ and other polysulfides in the form of the surface double layer. The locally enriched interface-absorbed $\mathrm{Na}$ can further react with $\mathrm{Na}_{2} \mathrm{~S}_{x}$, resulting in the formation of the final product $\mathrm{Na}_{2} \mathrm{~S}$. The unique Co-S-Na molecular layer structure formed over the Co nanoparticles enables the rapid conversion of polysulfides to form the final $\mathrm{Na}_{2} \mathrm{~S}$ product during discharging process.

During charge, $\mathrm{Na}$ in $\mathrm{Co}-\mathrm{S}-\mathrm{Na}$ is more susceptible to dissociation than prior to $\mathrm{Na}$ in $\mathrm{Na}_{2} \mathrm{~S}_{x}$ molecule induced by the oxidation of sulfur anion and leads to fast kinetics (Fig. 5c III). The Na-deficient Co-S-Na will form with $\mathrm{Na}$ atoms being reduced and removed from the S-ML. The exposed $\mathrm{S}$ of the Na-deficient Co-S-Na can capture $\mathrm{Na}$ from $\mathrm{Na}_{2} \mathrm{~S}_{x}$ to fill the vacancies left behind by the removed $\mathrm{Na}$ atoms,

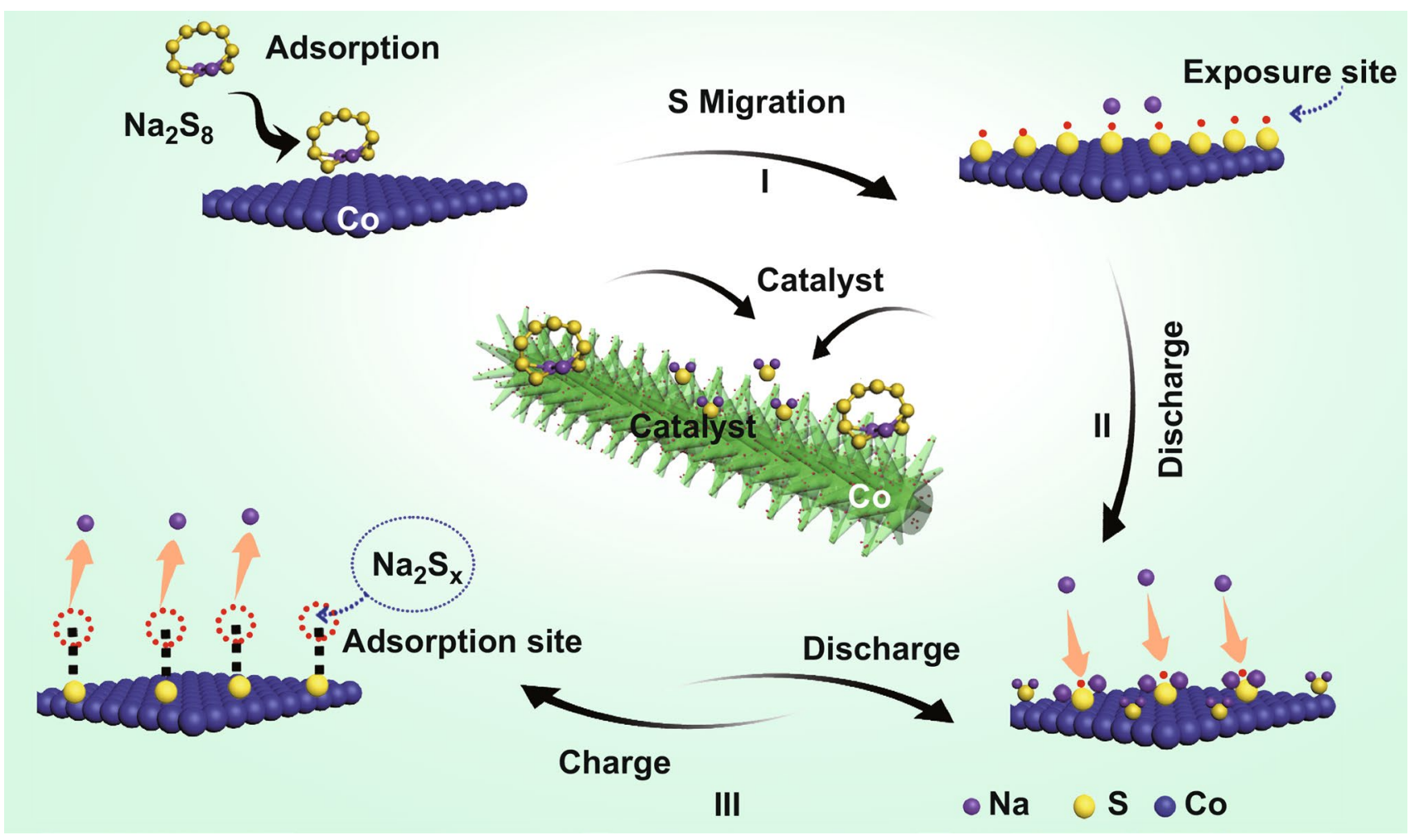

Fig. 5 Mechanism for enhancing the Na-S battery performance 
and a shorter chain polysulfide will be form the remainder of the $\mathrm{Na}_{2} \mathrm{~S}_{x}$. The Na capture reaction is mildly endothermic, in contrast to the highly endothermic Na reduction of $\mathrm{Na}_{2} \mathrm{~S}_{x}$, and therefore is viable under the charge condition. The captured $\mathrm{Na}$ can be further reduced and removed from Co-S-Na, which ends the old catalytic cycle and starts the new cycle. The Co nanoparticle hence exhibits catalytic performance by breaking the highly endothermic one-step $\mathrm{Na}$ reduction reaction of the polysulfides molecules into two mildly endothermic steps, as well as by facilitating the $\mathrm{Na}$ capture and oxidation with the S-ML. The consumption rate of electrons is likely to be much faster on Co nanoparticles than the consumption rate on the graphite surface. Such catalytic effects of Co nanoparticles under a large charging current are highly significant to their use in electrodes.

\section{Conclusion}

In summary, a CNF-L@Co/S material with "leaf-branch" structure proposed as a cathode for high performance $\mathrm{Na}-\mathrm{S}$ batteries. The unique "leaf-branch" structure ensures effective utilization due to Co "leaf" during electrocatalytic reactions and fast electron transfer due to the porous CNF "branch," which plays a key role in improving the overall electrochemical performance. As a sulfur host, the CNF-L@Co/S cathode exhibits a high reversible capacity of $1201 \mathrm{mAh} \mathrm{g}^{-1}$ at $0.1 \mathrm{C}$, stable long-term cycling stability and superior rate performance. The catalytic effect of Co nanoparticles on sodium polysulfides was verified by various spectroscopic measurements and DFT calculation. Results demonstrated that the $\mathrm{Co}-\mathrm{S}-\mathrm{Na}$ interface layer formed on the surface of Co can alter the pathway for the reduction reaction and promote fast kinetics. Overall, this work provides new insights for the rational design of high performance composite electrodes for RT Na-S batteries.

Acknowledgements This work is financially supported by Grants from the National Natural Science Foundation of China (No. 21773188, 21972111, U1530401), Natural Science Foundation of Chongqing (cstc2018jcyjAX0714). Furthermore, especially thanks to pan Liu, Longcheng Zhang, Longzhen Zhang, Wei Zhong, Dingyu Liu, Bingshu Guo, Tingting Yang, Jianhua Deng and Qianru Ma for their help in test aspects.

Open Access This article is licensed under a Creative Commons Attribution 4.0 International License, which permits use, sharing, adaptation, distribution and reproduction in any medium or format, as long as you give appropriate credit to the original author(s) and the source, provide a link to the Creative Commons licence, and indicate if changes were made. The images or other third party material in this article are included in the article's Creative Commons licence, unless indicated otherwise in a credit line to the material. If material is not included in the article's Creative Commons licence and your intended use is not permitted by statutory regulation or exceeds the permitted use, you will need to obtain permission directly from the copyright holder. To view a copy of this licence, visit http://creativecommons.org/licenses/by/4.0/.

Electronic Supplementary Material The online version of this article (https://doi.org/10.1007/s40820-020-00563-6) contains supplementary material, which is available to authorized users.

\section{References}

1. J. Goodenough, How we made the Li-ion rechargeable battery. Nat. Electron. 1, 204-204 (2018). https://doi.org/10.1038/s4192 8-018-0048-6

2. M. Shi, S. Zhang, Y. Jiang, Z. Jiang, L. Zhang et al., Sandwiching sulfur into the dents between N, O Co-doped graphene layered blocks with strong physicochemical confinements for stable and high-rate Li-S batteries. Nano-Micro Lett. 12, 146 (2020). https://doi.org/10.1007/s40820-020-00477-3

3. Y. Wang, B. Zhang, W. Lai, Y. Xu, S. Chou et al., A comprehensive review on research progress and cell chemistry. Adv. Energy Mater. 7, 1602829 (2017). https://doi.org/10.1002/aenm.20160 2829

4. P. Adelhelm, P. Hartmann, C. Bender, M. Busche, C. Eufinger et al., From lithium to sodium: cell chemistry of room temperature sodium-air and sodium-sulfur batteries. Beilstein. J. Nanotech. 6, 1016-1055 (2015). https://doi.org/10.3762/bjnano.6.105

5. J. Kim, Y. Lee, S. Cho, J. Gwon, H. Cho et al., Nanomat Li-S batteries based on all-fibrous cathode/separator assemblies and reinforced Li metal anodes: towards ultrahigh energy density and flexibility. Energy Environ. Sci. 12, 177-186 (2019). https://doi. org/10.1039/C8EE01879K

6. D. Liu, W. Li, Y. Zheng, Z. Cui, X. Yan et al., In situ encapsulating $\alpha$-MnS into N, S-Co doped nanotube-like carbon as advanced anode material: $\alpha \rightarrow \beta$ phase transition promoted cycling stability and superior Li/Na-storage performance in half/full cells. Adv. Mater. 30, 1706317 (2018). https://doi.org/10.1002/adma.20170 6317

7. D. Gueon, J. Hwang, S. Yang, E. Cho, K. Sohn et al., Spherical macroporous carbon nanotube particles with ultrahigh sulfur loading for lithium-sulfur battery cathodes. ACS Nano 12, 226-2339 (2018). https://doi.org/10.1021/acsnano.7b05869

8. T. Yang, W. Gao, B. Guo, R. Zhan, Q. Xu et al., A railway-like network electrode design for room temperature Na-S battery. J. Mater. Chem. A 7, 150-156 (2019). https://doi.org/10.1039/ c8ta09556f

9. Y. Wang, W. Lai, Y. Wang, S. Chou, X. Ai et al., Sulfurbased electrodes that function via multielectron reactions for 
room-temperature sodium-ion storage. Angew. Chem. Int. Ed. 58, 18324-18337 (2019). https://doi.org/10.1002/ange.20190 2552

10. Y. Wang, W. Lai, S. Chou, H. Liu, S. Dou, Remedies for polysulfide dissolution in room-temperature sodium-sulfur batteries. Adv. Mater. 32, 1903952 (2020). https://doi.org/10.1002/ adma.201903952

11. A. Ghosh, S. Shukla, M. Monisha, A. Kumar, B. Lochab et al., Sulfur copolymer: a new cathode structure for room-temperature sodium-sulfur batteries. ACS Energy Lett. 2, 2478-2485 (2017). https://doi.org/10.1021/acsenergylett.7b00714

12. G. Xia, L. Zhang, X. Chen, Y. Huang, D. Sun et al., Carbon hollow nanobubbles on porous carbon nanofibers: an ideal host for high-performance sodium-sulfur batteries and hydrogen storage. Energy Storage Mater. 14, 314-323 (2018). https://doi. org/10.1016/j.ensm

13. S. Wei, S. Xu, A. Agrawral, S. Choudhury, Y. Lu et al., A stable room-temperature sodium-sulfur battery. Nat. Commun. 7, 11722 (2016). https://doi.org/10.1038/ncomms 11722

14. C. Fan, S. Liu, H. Li, Y. Shi, H. Wang et al., Synergistic mediation of sulfur conversion in lithium-sulfur batteries by a Gerber tree-like interlayer with multiple components. J. Mater. Chem. A 5, 11255-11262 (2017). https://doi.org/10.1039/c7ta02231j

15. B. Zhang, T. Sheng, Y. Liu, Y. Wang, L. Zhang et al., Atomic cobalt as an efficient electrocatalyst in sulfur cathodes for superior room-temperature sodium-sulfur batteries. Nat. Commun. 9, 4082 (2018). https://doi.org/10.1021/jacs.8b12973

16. X. Xu, D. Zhou, X. Qin, K. Lin, F. Kang et al., A room-temperature sodium-sulfur battery with high capacity and stable cycling performance. Nat. Commun. 9, 3870 (2018). https:// doi.org/10.1002/aenm.201602829

17. D. Liu, C. Zhang, G. Zhou, W. Lv, G. Ling et al., Catalytic effects in lithium-sulfur batteries: promoted sulfur transformation and reduced shuttle effect. Adv. Sci. 5, 1700270 (2018). https://doi. org/10.1002/advs.201700270

18. H. Lin, S. Zhang, T. Zhang, H. Ye, Q. Yao et al., Simultaneous cobalt and phosphorous doping of $\mathrm{MoS}_{2}$ for improved catalytic performance on polysulfide conversion in lithium-sulfur batteries. Adv. Energy Mater. 9, 1902096 (2019). https://doi. org/10.1002/aenm.201902096

19. J. Zhang, G. Li, Y. Zhang, W. Zhang, X. Wang et al., Vertically rooting multifunctional tentacles on carbon scaffold as efficient polysulfide barrier toward superior lithium-sulfur batteries. Nano Energy 64, 103905 (2019). https://doi.org/10.1016/j.nanoe n.2019.103905

20. Y. Zhan, A. Buffa, L. Yu, Z.C. Xu, D. Mandler, Electrodeposited sulfur and $\mathrm{Co}_{\mathrm{x}} \mathrm{S}$ electrocatalyst on buckypaper as high-performance cathode for Li-S batteries. Nano-Micro Lett. 12, 141 (2020). https://doi.org/10.1007/s40820-020-00479-1

21. L. Zhang, X. Chen, F. Wan, Z. Niu, Y. Wang et al., Enhanced electrochemical kinetics and polysulfide traps of indium nitride for highly stable lithium-sulfur batteries. ACS Nano 12, 95789586 (2018). https://doi.org/10.1021/acsnano.8b05466

22. N. Zheng, G. Jiang, X. Chen, J. Mao, N. Jiang et al., Battery separators functionalized with edge-rich $\mathrm{MoS}_{2} / \mathrm{C}$ hollow microspheres for the uniform deposition of $\mathrm{Li}_{2} \mathrm{~S}$ in high-performance lithium-sulfur batteries. Nano-Micro Lett. 11, 43 (2019). https ://doi.org/10.1021/jacs.7b11434

23. S. Zheng, P. Han, Z. Han, P. Li, H. Zhang et al., Nano-copper-assisted immobilization of sulfur in high-surface-area mesoporous carbon cathodes for room temperature Na-S batteries. Adv. Energy Mater. 4, 140022625 (2014). https://doi. org/10.1002/aenm.201400226

24. N. Wang, Y. Wang, Z. Bai, Z. Fang, X. Zhang et al., High-performance room-temperature sodium-sulfur battery enabled by electrocatalytic sodium polysulfides full conversion. Energy. Environ. Sci. 13, 562-570 (2020). https://doi.org/10.1039/ C9EE03251G

25. S. Zhang, T. Pollard, X. Feng, O. Borodin, K. Xu et al., Altering the electrochemical pathway of sulfur chemistry with oxygen for high energy density and low shuttling in a $\mathrm{Na} / \mathrm{S}$ battery. ACS Energy Lett. 5, 1070-1076 (2020). https://doi.org/10.1021/acsen ergylett.9b02746

26. Z. Du, X. Chen, W. Hu, C. Chuang, S. Xie et al., Cobalt in nitrogen-doped graphene as single-atom catalyst for high-sulfur content lithium-sulfur batteries. J. Am. Chem. Soc. 141, 3977-3985 (2019). https://doi.org/10.1021/jacs.8b12973

27. B. Guo, W. Du, T. Yang, J. Deng, D. Liu et al., Nickel hollow spheres concatenated by nitrogen-doped carbon fibers for enhancing electrochemical kinetics of sodium-sulfur batteries. Adv. Sci. 7, 1902617 (2020). https://doi.org/10.1002/advs.20190 2617

28. Y. Tian, Y. An, C. Wei, B. Xi, S. Xiong et al., Flexible and freestanding $\mathrm{Ti}_{3} \mathrm{C}_{2} \mathrm{~T}_{\mathrm{x}}$ MXene@ $\mathrm{Zn}$ paper for dendrite-free aqueous zinc metal batteries and nonaqueous lithium metal batteries. ACS Nano 13, 11676-11685 (2019). https://doi.org/10.1021/ acsnano.9b05599

29. Y. Hu, D. Ye, B. Luo, H. Hu, X. Zhu et al., A binder-free and free-standing cobalt sulfide@ carbon nanotube cathode material for aluminum-ion batteries. Adv. Mater. 30, 1703824 (2018). https://doi.org/10.1002/adma.201703824

30. K. He,T. Tsegaye Tsega, X. Liu, J. Zai, X. Li et al., Utilizing the space-charge region of the FeNi-LDH/CoP p-n junction to promote performance in oxygen evolution electrocatalysis. Angew. Chem. Int. Ed. 58, 11903-11909 (2019). https://doi.org/https:// doi.org/10.1002/anie.201905281

31. D. Ma, B. Hu, W. Wu, X. Liu, J. Zai et al., Highly active nanostructured $\mathrm{CoS}_{2} / \mathrm{CoS}$ heterojunction electrocatalysts for aqueous polysulfide/iodide redox flow batteries. Nat. Commun. 10, 3367 (2019). https://doi.org/10.1038/s41467-019-11176-y

32. Q. Lu, J. Yu, X. Zou, K. Liao, P. Tan et al., Self-catalyzed growth of $\mathrm{Co}, \mathrm{N}$-codoped CNTs on carbon-encased $\mathrm{CoS}_{\mathrm{x}}$ surface: a noble-metal-free bifunctional oxygen electrocatalyst for flexible solid Zn-air batteries. Adv. Funct. Mater. 29, 1904481 (2019). https://doi.org/10.1002/adfm.201904481

33. J. Liu, W. Zhang, Y. Chen, P. Zhou, K. Zhang, A novel biomimetic dandelion structure-inspired carbon nanotube coating with sulfur as a lithium-sulfur battery cathode. Nanotechnology 30, 155401 (2019). https://doi.org/10.1088/1361-6528/aafe46

34. Y. Zhang, J. Zai, K. He, X. Qian, $\mathrm{Fe}_{3} \mathrm{C}$ nanoparticles encapsulated in highly crystalline porous graphite: salt-template synthesis and enhanced electrocatalytic oxygen evolution activity 
and stability. Chem. Commun. 54, 3158 (2018). https://doi. org/10.1039/c8cc01057a

35. K. He, J. Zai, X. Liu, Y. Zhu, A. Iqbala et al., One-step construction of multi-doped nanoporous carbon-based nanoarchitecture as an advanced bifunctional oxygen electrode for Zn-Air batteries. Appl. Catal. B-Environ. 265, 118594 (2020). https://doi. org/10.1016/j.apcatb.2020.118594

36. D. Ji, S. Peng, L. Fan, L. Li, X. Qin et al., Thin $\mathrm{MoS}_{2}$ nanosheets grafted MOFs-derived porous $\mathrm{Co}-\mathrm{N}-\mathrm{C}$ flakes grown on electrospun carbon nanofibers as self-supported bifunctional catalysts for overall water splitting. J. Mater. Chem. A 5, 23898-23908 (2017). https://doi.org/10.1039/c7ta08166a

37. L. Zhang, H. Chen, G. Hou, L. Zhang, Q. Li et al., Puzzleinspired carbon dots coupled with cobalt phosphide for constructing a highly-effective overall water splitting interface. Chem. Commun. 56, 257-260 (2020). https://doi.org/10.1039/ c9cc08032e

38. L. Fan, R. Ma, Y. Yang, S. Chen, B. Lu, Covalent sulfur for advanced room temperature sodium-sulfur batteries. Nano Energy 28, 304-310 (2016). https://doi.org/10.1016/j.nanoen

39. J. Wang, G. Yang, J. Chen, Y. Liu, Y. Wang et al., Flexible and high-loading lithium-sulfur batteries enabled by integrated three-in-one fibrous membranes. Adv. Energy Mater. 9, 1902001 (2019). https://doi.org/10.1002/aenm.201902001

40. C. Dai, J. Lim, M. Wang, L. Hu, Y. Chen et al., Honeycomblike spherical cathode host constructed from hollow metallic and polar $\mathrm{Co}_{9} \mathrm{~S}_{8}$ tubules for lithium-sulfur batteries. Adv. Funct. Mater. 28, 1704443 (2018). https://doi.org/10.1002/adfm.20170 4443

41. R. Carter, L. Oakes, A. Douglas, N. Muralidharan, A.P. Cohn et al., A sugar-derived room-temperature sodium sulfur battery with long term cycling stability. Nano Lett. 17, 1863-1869 (2017). https://doi.org/10.1021/acs.nanolett.6b05172

42. Q. Lu, X. Wang, J. Cao, C. Chen, K. Chen et al., Freestanding carbon fiber cloth/sulfur composites for flexible room-temperature sodium-sulfur batteries. Energy Storage Mater. 8, 77-84 (2017). https://doi.org/10.1016/j.ensm
43. Y. Wang, J. Yang, W. Lai, S. Chou, Q. Gu et al., Achieving highperformance room-temperature sodium-sulfur batteries with S@ interconnected mesoporous carbon hollow nanospheres. J. Am. Chem. Soc. 138, 16576-16579 (2016). https://doi.org/10.1021/ jacs.6b08685

44. Q. Ma, G. Du, B. Guo, W. Tang, Y. Li et al., Carbon-wrapped cobalt nanoparticles on graphene aerogel for solid-state roomtemperature sodium-sulfur batteries. Chem. Eng. J. 388, 124210 (2020). https://doi.org/10.1016/j.cej.2020.124210

45. W. Du, W. Gao, T. Yang, B. Guo, L. Zhang et al., Cobalt nanoparticles embedded into free-standing carbon nanofibers as catalyst for room-temperature sodium-sulfur batteries. J. Colloid. Interface Sci. 565, 63-69 (2020). https://doi.org/10.1016/j. jcis.2020.01.010

46. K. He, J. Zai, X. Liu, Y. Zhu, A. Iqbala et al., One-step construction of multi-doped nanoporous carbon-based nanoarchitecture as an advanced bifunctional oxygen electrode for $\mathrm{Zn}$-Air batteries. Appl. Cataly. B-Environ. 265, 118594 (2020). https://doi. org/10.1016/j.apcatb

47. T. Yang, B. Guo, W. Du, K. Aslam, T. Tao et al., Design and construction of sodium polysulfides defense system for roomtemperature Na-S battery. Adv. Sci. 6, 1901557 (2019). https:// doi.org/10.1002/advs.201901557

48. W. Zhu, A. Paolella, C. Kim, D. Liu, Z. Feng et al., Investigation of the reaction mechanism of lithium sulfur batteries in different electrolyte systems by in situ Raman spectroscopy and in situ X-ray diffraction. Sustain. Energy Fuels 1, 737-747 (2017). https ://doi.org/10.1039/C6SE00104A

49. Q. Li, Y. Song, R. Xu, L. Zhang, J. Gao et al., Biotemplating growth of nepenthes-like $\mathrm{N}$-doped graphene as a bifunctional polysulfide scavenger for Li-S batteries. ACS Nano 12, 10240 10250 (2018). https://doi.org/10.1021/acsnano.8b05246

50. L. Zhou, L. Yao, S. Li, J. Zai, S. Li et al., The combination of intercalation and conversion reactions to improve the volumetric capacity of the cathode in Li-S batteries. J. Mater. Chem. A 7, 3618 (2019). https://doi.org/10.1039/c8ta11375k 